\title{
Percepción y autopercepción de los estereotipos de género en estudiantes universitarios de la región de Valparaíso a través de la publicidad
}

\section{Perception and self-perception of gender stereotypes in university students at Valparaíso region through advertising}

\author{
Lila Farías Muñoz y Varinia Cuello Riveros ${ }^{1}$ \\ Recibido: 08-09-2017 - Aceptado: 12-12-2017 \\ DOI: https://doi.org/10.26441/RC17.1-2018-A8
}

\begin{abstract}
RESUMEN: Esta investigación, se enmarca en la línea de integración e inclusión social de la Universidad Viña del Mar, y determina la concepción que poseen los jóvenes universitarios de primer año de la región de Valparaíso - Chile, sobre los estereotipos de género reflejados en la sociedad y la publicidad. Y dentro de ésta, tanto la publicidad en general como de las universidades de la región. Este estudio es de corte cualitativo y en él, los jóvenes reconocen la persistencia del machismo, así como la aparición de un nuevo estereotipo femenino que exige del hombre un cambio. Los modelos de hombre y mujer que presenta la sociedad y la publicidad en general, son los tradicionales. En la publicidad de las universidades, los estudiantes reconocen mayores rasgos de alteridad. Esta mirada, permite observar las coincidencias o diferencias en relación a su visión personal, y su incidencia en el desarrollo de estrategias de comunicación.
\end{abstract}

Palabras claves: género; estereotipos; sociedad; publicidad; jóvenes.

ABSTRACT: This research is part of the line of integration and social inclusion of the Viña del Mar University, and determines the conception that young first-year university students from the region of Valparaíso (Chile) have, about the gender stereotypes reflected in society and advertising. Advertising considers both advertising in general and that of regional universities. This study is qualitative and in it, young people recognize the persistence of machismo, as well as the appearance of a new feminine stereotype that demands of man a change. The models of men and women presented by the society and advertising in general are traditional. In university advertising, students recognize greater features of otherness. This view allows observing the coincidences or differences in relation to their personal vision, and its incidence in the development of communication strategies.

Key words: gender; stereotypes; students; society; advertising; youth.

\footnotetext{
Lila Farías Muñoz es Doctora en Ciencias de la Información de la Universidad Complutense de Madrid y Licenciada en Comunicación Social de la Universidad Central de Venezuela. Especialista en Ética de la comunicación y empresarial, Comunicación corporativa y Asuntos públicos. Actualmente es Directora de la Escuela de Comunicaciones de la Universidad Viña del Mar. Ifarias@uvm.cl, https://orcid.org/0000-0003-3042-5774

Varinia Cuello Riveros es Periodista y Magíster en Comunicación por la Universidad Diego Portales (Chile), Master en Comunicación Empresarial por la Universidad Pompeu Fabra (España). Es profesora en la Escuela de Comunicaciones en Universidad Viña del Mar. vcuello@uvm.cl, https://orcid.org/0000-0003-2155-8073
} 


\section{Introducción y estado de la cues- tión}

Para la Universidad Viña del Mar, la identidad de género es un tema que merece atención en el marco de sus valores de inclusión e igualdad, de allí la relevancia de profundizar en la percepción y autopercepción de género de los estudiantes universitarios de la región de Valparaíso (Chile) a través de la publicidad. En este sentido, a nivel nacional, el tema es de interés especialmente a partir del año 2015, debido a la campaña lanzada por el Gobierno de Michelle Bachelet, a través de los medios de difusión masiva promoviendo un cambio cultural para erradicar la discriminación de la mujer en la sociedad, así como la reciente aprobación por parte del senado de la Ley de Identidad de Género, el día 14 de junio de 2017.

La educación universitaria en la que se incorporó la mujer en 1877 , ha sido un fuerte símbolo de la igualdad entre géneros, la construcción de esta igualdad, tiene que ver con el logos, el pensamiento, a través del cual trasciende el individuo a otras esferas del quehacer en la sociedad, y en el que se construyen y refuerzan modelos a través de las distintas herramientas que posee la comunicación.

Esta es la primera etapa de una investigación mayor que implicará, luego de esta fase cualitativa, la confirmación de los hallazgos con técnicas de tipo cuantitativo. Se inicia con un primer cuestionamiento: ¿qué relación existe entre la percepción que tienen los jóvenes acerca de los estereotipos de género considerando diferentes contextos, en primer término: la sociedad, la publicidad en general y la publicidad de las universidades de la región de Valparaíso; y como segundo aspecto, la percepción individual que tienen los jóvenes estudiantes al respecto? De aquí, que se asuma que la concepción de los estereotipos de géneros está enraizada en la sociedad y la cultura; y específicamente en la publicidad como una expresión de la sociedad misma. Por otra parte, se considera que los estereotipos de género que se asumen individualmente, responden de manera coherente con lo pautado por la sociedad.

Sobre la base de las percepciones y creencias profundas de los jóvenes en relación a los estereotipos de género, este estudio también pretende iluminar la construcción de la comunicación desde las universidades de la región, considerado un elemento estratégico en el fortalecimiento del vínculo de la institución con sus miembros, de tal manera que respete la cosmovisión y ética de los estudiantes, para promover instancias de diálogo y encuentro de los géneros dentro del quehacer universitario como social, haciendo énfasis en el uso de un lenguaje más adherido a su naturaleza. Este estudio no pretende hacer un análisis de la comunicación, que no sea más allá de identificar estereotipos de género en la publicidad de las universidades seleccionadas. Se busca que el conocimiento que genere esta investigación, sirva de base para crear comunicaciones y diálogos más relevantes con los jóvenes, así como para emprender con más 
certeza, la segunda fase de este proyecto, que es cuantitativa.

Ahora bien, al referirnos a género estamos hablando primero de biología, la diferencia de sexo. También estamos hablando de antropología, el ser humano dentro del contexto social y cultural. Aquí abordamos el concepto de estereotipos, donde la cultura determina la manera de cómo debe comportarse el individuo en sociedad y en relación con un otro.

La evolución de las percepciones de los estudiantes, hacia "una mayor sensibilización hacia la igualdad" (Mosteiro y Porto, 2017) es congruente con este estudio, considerando que el componente prescriptivo (Barberá y Martínez, 2004), de un estereotipo que está sostenido por la estructura social y que "es la representación que cada cual se plantea de cómo los demás piensan que debe comportarse" (p.58). Es así, como del hombre y de la mujer existe una amplia herencia cultural que ha moldeado la forma en que estos deben comportarse dentro de su entorno. Es decir, familia y sociedad ayudan en la construcción del estereotipo de género que a cada uno le toca representar en el mundo, y es en el vínculo con los otros donde esa construcción se reafirma o modifica.

Por otra parte, los estudios de género han enfatizado hasta ahora el tema de la desigualdad de la mujer en relación al hombre, es decir, el énfasis ha estado en la mujer. Quizás se deba a que el hombre desde siempre ha manejado las estructuras de poder, por tanto se ha colocado en un orden superior a la mujer y ha sido esta última la que he tenido que emprender la lucha por la igualdad de género.

Los estereotipos de género se componen de una serie de características que se asocian más a hombres o a mujeres. Los hombres asociados a roles de poder y las mujeres al desarrollo de trabajos de cuidado a los demás. Así, se asocia el poder en el nivel público a los hombres y el nivel privado a las mujeres (López-Zafra et al., 2008).

Es evidente que algo está cambiando en la sociedad actual, pero, a pesar de que las relaciones intergrupales entre los géneros están evolucionando, todavía persisten ciertos estereotipos de género que se resisten al cambio (Mosteiro y Porto 2017).

Las identidades masculinas y femeninas están social e históricamente constituidas, y en consecuencia, están sujetas a las miserias y a los vasallajes de la cultura patriarcal, pero también abiertas a las utopías del cambio y de la igualdad (Lomas 2005, p.263).

Aun cuando se pudieran mantener ciertos substratos culturales bastante primitivos en relación al juego de roles de los géneros, estos han ido evolucionando a través del tiempo y ha sido la publicidad, la literatura y los distintos medios de comunicación masiva, los exponentes de estos cambios en el tiempo. No podríamos decir que la publicidad genera estereotipos, se considera más bien que estos han sido generados a través de la cultura particular de cada sociedad y de cada individuo, como producto de las demandas de su proceso de adaptación al entorno 
(familia, escuela, sociedad) y lo que hace la publicidad son representaciones de estos estereotipos y al hacerlo pudiera provocar proyecciones e identificaciones del individuo con los mismos. En este sentido, el arquetipo de la alteridad que "es capaz de dar a todas las polaridades derechos iguales, en un espectro que va desde la oposición radical de los polos hasta su igualdad" (Byington, 2009, p.225), emerge con intensidad en los resultados de este estudio.

Este trabajo tiene por objetivo determinar los estereotipos de género de los y las jóvenes universitarios de la región de Valparaíso de Chile, y su relación con la publicidad de las universidades y la publicidad en general; lo que permite también determinar el nivel de identificación de los y las jóvenes con las tipologías predefinidas de los géneros; establecer el nivel de asociación de las tipologías predefinidas de géneros de hombres y mujeres, con la publicidad de las universidades de la región de Valparaíso evaluadas en este estudio; y determinar los estereotipos subyacentes de los géneros, que refleja la sociedad chilena, desde la perspectiva de los y las jóvenes.

\section{Método}

La investigación que se realiza es de tipo cualitativo, descriptivo y transversal, tal como se define en el método de investigación científico social, comen- zando con una inmersión en el campo y a la luz de los resultados, surgen las hipótesis de investigación.

\section{Diseño y muestra}

Este estudio es de carácter cualitativo y de tipo descriptivo. Esta investigación cualitativa permitió el levantamiento de la información. Se desarrollaron entrevistas en profundidad y focus group, diseñando para ello, un guión de tópicos que cubrió con amplitud los objetivos. Los discursos planteados por los estudiantes de primer año de las universidades de la región de Valparaíso, a través de estos instrumentos, son la fuente principal del análisis e interpretación que fundamenta este estudio.

La publicidad de las universidades de la región de Valparaíso ${ }^{2}$ seleccionada fue la publicada en las páginas web de dichas instituciones como promoción para la captación de estudiantes para el periodo 2016. Para explorar los estereotipos de la publicidad en general, se les preguntó abiertamente al respecto y también se validó con imágenes que ellos mismos escogieron de una muestra de revistas que se comercializan en el mercado chileno, con diferentes enfoques (tradicional, moda, dirigidas específicamente a la mujer o a los hombres) y que fueron consideradas para el estudio. La muestra de revistas presentada fue la misma para todos los participantes.

2 Universidades consideradas en el estudio: Universidad de Valparaíso (UV), Pontificia Universidad Católica de Valparaiso (PUCV), Universidad de Playa Ancha (UPLA), Universidad Técnica Federico Santa María (UTFSM), Universidad Andrés Bello (UNAB) y Universidad de Viña del Mar (UVM) 
El universo muestral estuvo conformado por estudiantes universitarios de las cinco (5) principales universidades de la región de Valparaíso, según ranking de matrícula, corte 2016, es decir, en el primer año o semestre de la carrera, publicado por Consejo Nacional de Educación de Chile (CNDE), donde la mitad eran hombres y la otra mitad, mujeres. Los sujetos fueron seleccionados por conveniencia, según su dis- ponibilidad para el estudio. Se realizó un muestreo que se basó en la consulta a 20 jóvenes entre los 18 y 25 años de edad (10 mujeres y 10 varones) a través de entrevistas en profundidad. Se realizaron 4 grupos de discusión mixtos, compuestos por 8 jóvenes cada uno. En total, se realizó consulta a 52 jóvenes estudiantes universitarios. La muestra se cerró cuando se observó la saturación.

Cuadro 1. Especificación del guión de tópicos.

\begin{tabular}{|l|l|}
\hline Tópico 1 & Identificación: Descripción personal \\
\hline Tópico 2 & Percepción de género: Percepciones a cerca de las representaciones sociales. \\
\hline Tópico 3 & Percepción de género: Percepciones acerca de la publicidad en general. \\
\hline Tópico 4 & En relación a sí mismo: Imagen de sí mismo. \\
\hline Tópico 5 & $\begin{array}{l}\text { Percepción de género: Percepciones en relación a la publicidad de las } \\
\text { Universidades. Recordación y evaluación de la publicidad de Universidades. }\end{array}$ \\
\hline
\end{tabular}

Fuente: elaboración propia

\section{Análisis y Resultados}

\subsection{La construcción de estereotipos de género a partir de la cultura.}

El estudio arroja la percepción de que en la actualidad se habla tanto de "machismo" como de "feminismo", el primero relacionado con la tradicional visión preeminente del hombre; y el segundo, el feminismo, identificándolo como un síntoma de cambio del género femenino en la sociedad, reflejo del empoderamiento de la mujer ("el feminismo es la lucha por la igualdad de géneros y ganar nuestros propios espacios"; "ahora hay presidentas que son mujeres"), pero asegu- ran que aún predomina el hombre ("ocupan puestos de trabajo más bajos y con grandes brechas salariales"). Se reconoce en lo expresado por los estudiantes, la aparición del concepto "feminazi" de forma generalizada en hombres y mujeres, que implica aspectos de forma, como el cambio de la apariencia, rasgos en la forma de vestir, cortes de pelo, maquillaje o ausencia de este; y aspectos de fondo como la forma de vincularse con el entorno, actitudes plasmadas en la comunicación verbal y no verbal, verbalización de mensajes agresivos o displicentes, interpretación de los mensajes que provienen del entorno y la 
sociedad influimos también por esta postura ("es odiar y desprestigiar al hombre"; "se comportan como hombres, pero de manera grotesca").

Los resultados arrojan la idea de que en la sociedad se piensa que "la mujer es quien le da firmeza al hogar”, basado en el hecho de que la mujer es quien cría y le da sostén emocional al hogar, y se muestra como un reflejo de las experiencias de vida de los estudiantes, en sus familias o entornos cercanos. Respecto de la maternidad, las mujeres afirman que debido a que las mujeres se pueden embarazar, eso las estar en una posición desventajosa a la del hombre en relación a las conquistas laborales ("la maternidad inhibe el desarrollo laboral de la mujer, no te contratan para ciertos trabajos si eres mujer"). Así, al momento de optar por una persona para un puesto de trabajo ellas tienen la certeza que serán discriminadas por el solo hecho de ser mujeres.

Los y las jóvenes señalan que la sociedad cuestiona a la mujer cuando se trata de relaciones con el otro sexo ("si ella sale con varios hombres se le cuestiona"), lo que hace que nuevamente emerja la percepción desde las mujeres, de desigualdad frente a los hombres, ya que al hombre no se le enjuicia de esta manera ("sale con varias minas, es un macho"), sino que por el contrario se reconoce que se estimula en el hombre vincularse de manera temprana con las mujeres. Ellas señalan además, que la sociedad le ha impuesto a la mujer un estereotipo, con el cual la mayoría de ellas no está de acuerdo, asociado a la perfección, limpieza, cánones de belleza específicos que en general no tienen relación con la realidad, ni con la fisionomía de la generalidad de las mujeres ("la mujer debe ser perfecta, lucir bien, ser delgada, bella, limpia, educada, una señorita"). Sobre esto último se refieren a ciertos códigos morales de la mujer "decente" según la sociedad, que perciben se les imponen desde fuera de ellas ("una mujer que sale con varios hombres es una mala mujer, una mujer que exhibe demasiado su cuerpo en su forma de vestir es una mala mujer").

Hombres y mujeres jóvenes, a pesar de percibir ciertos cambios respecto de la visión del hombre, reconocen el carácter machista que le confiere la sociedad, esto implica que desde esta perspectiva se plantea como el género dominante en cuanto a lo físico, intelectual y económico; para las mujeres se trata de un machismo que inhibe las emociones del hombre, lo limita, coharta restringiéndolo permanentemente ("la sociedad exige que cumpla con su rol y si se sensibiliza se ve mal"). Sin embargo, los mismos varones destacaron la idea de que el hombre está perdiendo su rol en la sociedad, reconocen este cambio, pero no lo ven como algo positivo, sino que más bien identifican elementos que desde su propia perspectiva los perjudican en la imagen que proyectan ("se ha vuelto flojo" y "no tiene como serle leal al machismo"). Por su parte, las mujeres reconocen la tendencia del machismo, pero también observan que la posición del hombre se está debilitando, esto por varios aspectos mencionados, desde el debilitamiento del hombre en sus roles tradicionales de proveedor o más fuerte; hasta aspectos que tienen que ver 
con que dada una mayor visibilización de la mujer, el hombre se ve ocultado ("ya no es el sexo fuerte").

\subsection{Lo femenino y lo masculino desde la visión personal de los jóvenes.}

La percepción de empoderamiento de las mujeres, tanto en sus relaciones sociales como en su desempeño individual; y el dejar de ver a los hombres como superiores a ellas, se expone en las entrevistas aplicadas a las jóvenes. Ellas ven al hombre como partícipe de la dinámica social y familiar de una manera más igualitaria, e incluso en algunos aspectos, ellas se ven más empoderadas que ellos, llegando a ganarse espacios que antes no se pensaban ("más igualdad en trabajo, estudios, aporte económico, cuidado de los hijos y el hogar"; "las mujeres ahora con más poder, autonomía y haciéndose valer por su inteligencia"; "la mujer está luchando por su lugar en la sociedad y no tiene miedo de dar su opinión"). Un grupo de ellas ve al hombre como un buen compañero y en ese sentido lo incluyen en su cosmovisión del feminismo, los perciben más abiertos a dialogar, transar y construir relaciones igualitarias y aportativas, tanto para ellas como para ellos, dado que los hombres no serán frente a las mueres, sentirse amenazados por estos cambios ("hay hombres con ideologías femeninas"); otro grupo de mujeres afirman que la mujer no siempre necesita a un hombre, ellas perciben que primero está el reconocerse mujer, individuo, y luego el ser pareja con un hombre; entonces, el establecerse en pareja con un hombre no es percibido como una obligación, sino que como una posibilidad para la mujer ("el hombre va a ser una opción para la mujer"). Cuando se refieren a la igualdad, consideran que se aplica en el trabajo, lo económico, el desarrollo profesional y a las responsabilidades del hogar, pero no aplica en el ámbito sexual, ya que ellas mismas enjuician a sus pares cuando estas tienen una vida sexual muy activa y abierta, lo que no perciben como contradictorio ("una niña con muchos niños, es fácil, se ve mal"). En tanto que al referirse al hombre, si bien tampoco le justifican una vida libremente sexual y abierta, les parece que no es tan enjuiciado como la mujer, dando a entender que esto es más propio de lo masculino, a quien la sexualidad abierta y libre no lo rebaja ante la sociedad. Considerando lo anterior, se muestra una marcada significación en términos de la libertad sexual en los hombres, que parece imponer la superioridad en otros aspectos del desenvolvimiento de este género en relación a la mujer. Algunas jóvenes piensan que es la mujer quien cambiará el machismo en la sociedad, lo que se basaría en aspectos como el éxito y el poder, dado por dónde se sitúe la mujer socialmente, el trabajo que desempeñe o el dinero que posea ("cuando la mujer tiene éxito y gana más dinero, el machismo en el hombre baja”).

Los hombres reconocen el progreso de la mujer en la sociedad. Ellos piensan que actualmente las mujeres están más: "empoderadas", "aguerridas", "independientes", "mandan al hombre". En este sentido, ellos acotan que es bueno que la mujer valore al hombre, que si bien es cierto es positivo que las mujeres 
sean independientes y poderosas, puedan reconocer a los varones por su aporte. Ahora, para algunos de los hombres jóvenes entrevistados, las mujeres empoderadas chocan con su propio empoderamiento masculino, entran en conflicto esa fortaleza que los hombres reconocen en las mujeres, con la propia fortaleza que ven en ellos mismos (me cuesta lidiar con mujeres empoderadas porque yo también soy empoderado; hay roce). También reconocen que los hombres son más inmaduros que la mujer, en un aspecto global, y que a veces no saben lo que quieren, a diferencia de la mujer que parece ser más decidida en todo, más resuelta y sólida. Sin embargo, acotan que a pesar del empoderamiento de la mujer, el hombre en lo laboral llega más lejos ("siempre van a preferir a un hombre") y que es por esta razón que el hombre gana más dinero que las mujeres. Lo laboral es un ámbito que ellos reconocen, aún está dominado por los hombres.

Fue recurrente entre los hombres entrevistados, el comentario acerca de la maternidad como factor que rompe la equidad entre hombres y mujeres (cuando la mujer tiene hijos se rompe lo equitativo y la mujer debe estar en la casa", "Al tener los hijos ella se queda en casa y se rompe la equidad, el hombre es quien tiene que trabajar"). Para muchos es ésta una gran responsabilidad, se reconocen en su rol de proveedor y en conexión con el dinamismo patriarcal en sus aspectos benevolentes. En este momento en la relación hombre-mujer, ellos reconocen la importancia de su rol ("cuando ella tiene los hijos al hombre le toca asumir todo"). Por otra parte, tanto mujeres como hombres, consideran que la maternidad también como factor de discriminación laboral en esta sociedad ("la discriminan en los trabajos porque es madre potencial y en algún momento sale de licencia”).

\subsection{Estereotipos de género en la pu- blicidad en general}

Los y las jóvenes comentaron acerca de los diferentes estereotipos que destaca la publicidad en general en el contexto del país. Se refirieron al estereotipo de la mujer bonita, rubia, delgada y alta, de quienes piensan que son mujeres vacías, huecas. Viendo, además, este modelo como muy lejano al que se ve en su entorno cotidiano. Algunas mujeres, en un intento de justificar a este estereotipo de mujeres, se refieren a que es sólo su forma de ganarse la vida y que detrás de esa imagen de perfección, hay una personalidad distinta, con ideas e intenciones en la vida. En la investigación, a través de las respuestas de los jóvenes, también se identifica el estereotipo de la belleza masculina, que se refleja en hombres hermosos, de buen cuerpo, asociado al macho alfa, que le gusta estar rodeado de mujeres, pero en una actitud más contemplativa o narcisista.

Los y las universitarias identifican en las imágenes de mujeres entrenadas físicamente, fuerza de carácter, mujeres que pueden tomar decisiones, poner límites. Algunos de los hombres jóvenes entrevistados dicen que estas mujeres son temibles dadas sus características, con las que se sienten amenazados. Aparece en las respuestas de los estu- 
diantes, el estereotipo de la joven "talentosa" que refiere a la constancia de la mujer que busca alcanzar sus metas de manera diferente al hombre, si bien es cierto no expresan de manera específica las diferencias, se relacionan con el trabajo metódico y perseverante.

Los estudiantes identifican a un estereotipo de mujer objeto sexual, que se visualiza en la publicidad mostrando mucho desnudo y en relación al mismo, las jóvenes señalan que sólo le sirve a los propósitos del hombre, ellas no plantean ninguna opinión si se sienten motivadas a ser o actuar como este estereotipo, dado que sería este, el de la mujer "fácil" que se identifica en la sociedad. Para ambos géneros esta es una imagen de la mujer que la desvaloriza, que tergiversa su imagen y que no aporta nada ni en lo individual ni en lo social.

Finalmente está la mujer "auto realizada" que se muestra en una mujer madura, segura de sí misma, que ha alcanzado sus metas en la vida y es un estereotipo con el cual las jóvenes se sienten identificadas a futuro. Ellas creen que ese es su proyecto a seguir, de ahí que estudien y se perfeccionen.

\subsection{Estereotipos de género de la pu- blicidad de las universidades de la re- gión de Valparaíso.}

Tanto hombres como mujeres entrevistados pudieron asociar alguna publicidad con el estereotipo de la "mujer empoderada", y respecto a esta característica, los participantes de la investigación, hicieron escasa mención a la presencia de asimetrías entre los hombres y mujeres presentes en la publicidad. Por otra parte, los jóvenes valoraron en estas publicidades desarrolladas por las universidades de la región de Valparaíso el mensaje honesto, claro, que revele lo espontáneo y natural del quehacer estudiantil dentro de las universidades. Contrario a esto es lo artificial, lo falso de algunos rasgos observados en cierta publicidad, que aleja la empatía de ellos. Los jóvenes expresaron que la publicidad hace alusión a la educación y a la vida en común dentro de las casas de estudio de la región de Valparaíso.

Además, en las publicidades evaluadas, los jóvenes comentaron que el estereotipo que perciben es el de la "igualdad de géneros", que se refleja en "unión", "integración", "inclusión", "fraternidad" es decir, hay prácticamente ausencia de otros estereotipos. Estos aspectos fueron valorados de sobremanera por los participantes de la investigación.

\section{Conclusiones}

La sociedad y la publicidad en general, están asociados a estereotipos que se distancian de lo que los y las jóvenes conciben para sí mismos, en la construcción individual de cada uno. Sociedad y publicidad comercial son asociados a una imagen anticuada del hombre como machista y superior a la mujer, una visión tradicional de los varones. El estereotipo que emerge es una mujer empoderada, que asume el feminismo de manera incluyente con el hombre, se aleja esta visión del típico conflicto planteado considerando a la mujer feminista que entra en conflicto constante con el hombre. De igual 
manera, el hombre se orienta a una relación igualitaria de alteridad con la mujer, reconocen también un cambio en la percepción y vivencia de los hombres de su relación con las mujeres; encontrándose al mismo tiempo, la visión machista tradicional y esta nueva forma de relación, coexistiendo. Las respuestas de los jóvenes denotan el reconocimiento de que se trata de un proceso, aún en tránsito.

Los y las jóvenes consultados en esta investigación, consideran que la sociedad tiene una marcada tipificación de los géneros que no va en línea con la que ellos poseen. Por lo mismo no se ven reflejados en muchos aspectos en estas tipificaciones que desde otros perciben que se les imponen.

Algunas mujeres jóvenes se perciben a sí mismas con gran empoderamiento, sin sentir una superioridad masculina; un grupo valora a aquellos hombres que literalmente son capaces de ponerse en su lugar, ven al hombre como un buen compañero y en ese sentido lo incluyen en su cosmovisión del feminismo; otro grupo refieren que la mujer de hoy no necesita al hombre, esta postura se muestra en un extremo de las opiniones por ellos vertidas. Finalmente, algunas jóvenes piensan que es la mujer quien cambiará el machismo en la sociedad, usando el poder que da el dinero, ya no se habla aquí de igualdad, simplemente de modificar a través del poder del dinero.

Los y las jóvenes reflejan que las mujeres se han adaptado más fácilmente en la conquista del mundo masculino, pero pareciera que a los hombres se les puede hacer un poco más difícil. Ellos mismos expresan su perplejidad y escasos recursos de reacción antes las actuaciones de las mujeres en los contextos en que se desenvuelven. Algunas de las mujeres participantes de la investigación, intuyen esta dificultad del hombre, al observar que a ellos en variados contextos, se les tiene prohibido expresar sus emociones. En este sentido, es importante referir que ellas han observado hombres más sensibles y con ideologías femeninas.

El estereotipo de la mujer "decente" que no se permite tener múltiples relaciones, que no se "desnuda" socialmente para atraer al hombre, es un punto de coincidencia entre la sociedad y las mujeres jóvenes entrevistadas. Seguirá teniendo una fuerte crítica en la sociedad y en la publicidad comercial, la mujer fácil, con la cual las jóvenes no se sienten identificadas, dado lo anteriormente expuesto.

En relación a los estereotipos sociales y de la publicidad, que muestran a un hombre "alfa", atractivo, es posible que recoja la proyección de aquellos que se identifican con éste. Lo mismo ocurre con las mujeres flacas, rubias, bellas, altas. Aún cuando se piensa que son vacías, inconscientemente son un referente de la belleza femenina, aunque diste mucho con el prototipo común del país. Se refleja aquí lo coexistencia de estos estereotipos socioculturales femenino y masculino, con las propias autopercepciones de los y las jóvenes.

A la luz de la visión de los jóvenes en relación a los estereotipos de género, la publicidad comercial queda obsoleta, 
así como también les resulta retrograda la visión de la sociedad. En cuanto a la publicidad de las casas de estudio de la región de Valparaíso, se reconoce un mayor cuidado en ella, porque se encuentra un concepto más avanzado de la integración de los géneros entre los jóvenes estudiantes de la región, quienes son capaces de asimilar un "feminismo" y un "masculinismo" más incluyente e igualitario. Se destaca como elemento relevante el respeto mutuo.

\section{Bibliografía}

Aprile, O. (2003). La Publicidad puesta al día. Buenos Aires: La Crujía Ediciones.

Barberá, E. \& Martínez-Benlloch, I. (2004). Psicología y Género. Madrid: Pearson Educación, S.A.

Byington, C. (2009). Psicología Simbólica Junguiana: el viaje de humanización del cosmo en busca de la iluminación. Sao Paulo: Linear B.

Colom, J. (1997). Aportaciones al estudio de los estereotipos de género. Pedagogía social: revista interuniversitaria, 15-16, 145-154.

Farías, L. (2012). El contenido ético y empresarial en la identidad profesional del publicista chileno: ¿contradicción o complementariedad? (Tesis doctoral). Universidad Complutense de Madrid, España.

Gil, A. (2010). Estereotipos de género y sentido común dominante en la prensa gráfica mendocina, un análisis desde la intencionalidad editorial. Questión: revista especializada en periodismo y comunicación, 1, 27.

Guil, A. \& Gila J. (1999) La mujer actual en los medios, estereotipos cinematográficos. Revista Comunicar, 12, 89-93.

Jayme, M (1999). La identidad de género. Revista de Psicoterapia, 10(40), 5-22.

Jung, C. G. (1970). Arquetipos e inconsciente colectivo. Barcelona: Editorial Paidós.

Jung, C.G. (1970). Aion. Contribución a los simbolismos del sí-mismo. Buenos Aires: Editorial Paidós.

Lomas, C. (2005) ¿El otoño del patriarcado? El aprendizaje de la masculinidad y de la feminidad en la cultura de masas y la igualdad entre hombres y mujeres. Cuadernos de Trabajo Social 18, $259-258$.

López-Zafra E., García-Retamero R., Dieckmann A., Eagly A. (2008). Dinámica de estereotipos de género y poder: un estudio transcultural. Revista de Psicología Social, 23(2)

Melero Aguilar, N. (2010). Reivindicar la igualdad de mujeres y hombres en la sociedad: una aproximación al concepto de género. Barataria, Revista Castellano-Manchega de Ciencias Sociales, (11), 73-83.

Mosteiro M. \& Porto A. (2017) Análisis de los estereotipos de género en alumnado de formación profesional: diferencias según sexo, edad y grado. Revista de Investigación Educativa, 35(1), 151-165.

Segarra, M. \& Caraví, À. (2000). Nuevas masculinidades. Barcelona: Editorial Icaria. 